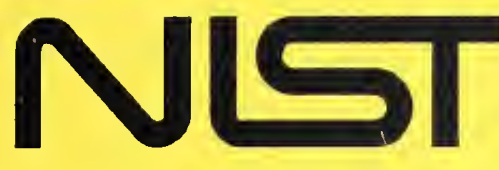

United States Department of Commerce Technology Administration

National Institute of Standards and Technology

NISTIR 5001

SHIELDED OPEN-CIRCUITED SAMPLE HOLDERS FOR DIELECTRIC AND MAGNETIC MEASUREMENTS OF LIQUIDS AND POWDERS

James Baker-Jarvis

Michael D. Janezic

Robert B. Stafford

$-Q C$

100

.056

\#5001

1993 



\section{SHIELDED OPEN-CIRCUITED SAMPLE HOLDERS FOR DIELECTRIC AND MAGNETIC MEASUREMENTS OF LIQUIDS AND POWDERS}

James Baker-Jarvis Michael D. Janezic

Electromagnetic Fields Division Electronics and Electrical Engineering Laboratory National Institute of Standards and Technology Boulder, Colorado 80303-3328

Robert B. Stafford

Department of Electrical Engineering Virginia Polytechnic Institute and State University Blackburg, Virginia 24061

March 1993

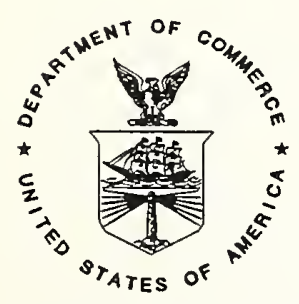




\begin{abstract}
This report overviews shielded open-circuit measurements and presents a comprehensive uncertainty analysis. We use a dominant-mode scattering formulation to develop an expression for the reflection coefficient in terms of bead and sample parameters. The formulation developed here eliminates the transformation through the various sections of the sample holder. We also extend the formulation to include magnetic measurements. The uncertainty analysis indicates a decrease in relative uncertainty with increasing sample length and with increasing frequency. The real part of the permittivity at low frequencies is very sensitive to measured phase of the reflection coefficient and sample length. The imaginary part of the permittivity of low-loss materials is not extremely sensitive to the sample length. For high-loss materials both the real and the imaginary parts of the permittivity are sensitive to the sample lengths. The minima of the reflection coefficient for low-loss materials occur at $n \lambda_{m} / 2$, where $n=1,2,3 \ldots$ and $\lambda_{m}$ is the wavelength in the material. These minima in the reflection coefficient correspond to regions of minimum uncertainty for the real part of the permittivity. The minimum uncertainty for low-loss materials occurs at frequencies where there is maximal interaction of the fields with the sample.
\end{abstract}

Key words: calibration; coaxial line; dielectric constant; liquids; loss factor; microwave measurements; open circuit; permeability measurement; permittivity measurement; powders; reflection method; transmission; uncertainty; waveguide 


\section{Contents}

1 Background 1

2 Theoretical Formulation 3

$\begin{array}{lll}2.1 & \text { Numerical Results } & 7\end{array}$

2.2 Magnetic Measurements 9

2.3 Characterization of the Sample Holder 11

$\begin{array}{lll}3 & \text { Uncertainty Analysis } & 11\end{array}$

4 Discussion $\quad 20$

5 References $\quad 20$

Appendix: Field Analysis of the Shielded Open Circuit 22 


\section{List of Figures}

1.1 The dielectric sample holder with grooves in bead section. . . . . . . . 1

1.2 The dielectric sample holder for an uncompensated bead. . . . . . . . 2

2.1 The magnitude of the reflection coefficient as a function of normalized length with $\epsilon_{R(3)}^{*}=(20,-0.01) . \ldots \ldots \ldots \ldots \ldots \ldots$

2.2 The real part of the permittivity of air as a function of frequency (maximum rms uncertainty over the band is $\left.\Delta \epsilon_{R(3)}^{\prime} \pm 0.03\right) \ldots \ldots \ldots . \ldots 9$

2.3 The real part of the permittivity of carbonaceous sand mixture as a function of frequency and water volume fraction (maximum rms uncertainty $\Delta \epsilon_{R(3)}^{\prime} \pm$ $0.35) \ldots \ldots \ldots \ldots \ldots \ldots \ldots \ldots \ldots \ldots \ldots$

2.4 The loss of carbonaceous sand mixture as a function of frequency and water volume fraction (maximum rms uncertainty $\Delta \epsilon_{R}^{\prime \prime} \pm 0.15$ ) . . . . . 10

3.1 The derivative of $\epsilon_{R(3)}^{\prime}$ with respect to $|\Gamma|$ with $\epsilon_{R(3)}^{*}=(5,-0.01) \ldots \ldots 15$

3.2 The derivative of $\epsilon_{R(3)}^{\prime \prime}$ with respect to $\theta_{11}$ with $\epsilon_{R(3)}^{*}=(5,-0.01) \ldots \ldots 15$

3.3 The derivative of $\epsilon_{R(3)}^{\prime}$ with respect to $L_{3} / \lambda_{m}$ with $\epsilon_{R(3)}^{*}=(5,-0.01)$. . . 16

3.4 The derivative of $\epsilon_{R(3)}^{\prime \prime}$ with respect to $L_{3} / \lambda_{m}$ with $\epsilon_{R(3)}^{*}=(5,-0.01) \ldots \ldots 16$

3.5 The relative uncertainty in $\epsilon_{R(3)}^{\prime}$ for a low-loss material as a function of normalized length with $\epsilon_{R(3)}^{*}=(5,-0.01)$ and $(20,-0.01) \ldots \ldots 17$

3.6 The relative uncertainty in $\epsilon_{R(3)}^{\prime \prime}$ for a low-loss material as a function of normalized length with $\epsilon_{R(3)}^{*}=(5,-0.01)$ and $(20,-0.01) \ldots \ldots 17$

3.7 The relative uncertainty in $\epsilon_{R(3)}^{\prime}$ for a high-loss material as a function of normalized length with $\epsilon_{R(3)}^{*}=(5,-10)$ and $(20,-20) \ldots \ldots 18$

3.8 The relative uncertainty in $\epsilon_{R(3)}^{\prime \prime}$ for a high-loss material as a function of normalized length with $\epsilon_{R(3)}^{*}=(5,-10)$ and $(20,-20) \ldots \ldots 18$

3.9 The relative uncertainty in $\epsilon_{R(2)}^{\prime}$ for the bead with $\epsilon_{R(2)}^{*}=(2.03,0)$ and for uncertainties in the bead permittivity of $\Delta \epsilon_{R(2)}^{\prime}=0.02$ and $\Delta \epsilon_{R(2)}^{\prime}=0$. 
3.10 The relative uncertainty in $\epsilon_{R(3)}^{\prime}$ as a function of frequency for permittivities $\epsilon_{R(3)}^{*}=(5,-0.01)$ and $\epsilon_{R(3)}^{*}=(20,-0.01) \ldots \ldots \ldots \ldots$ 


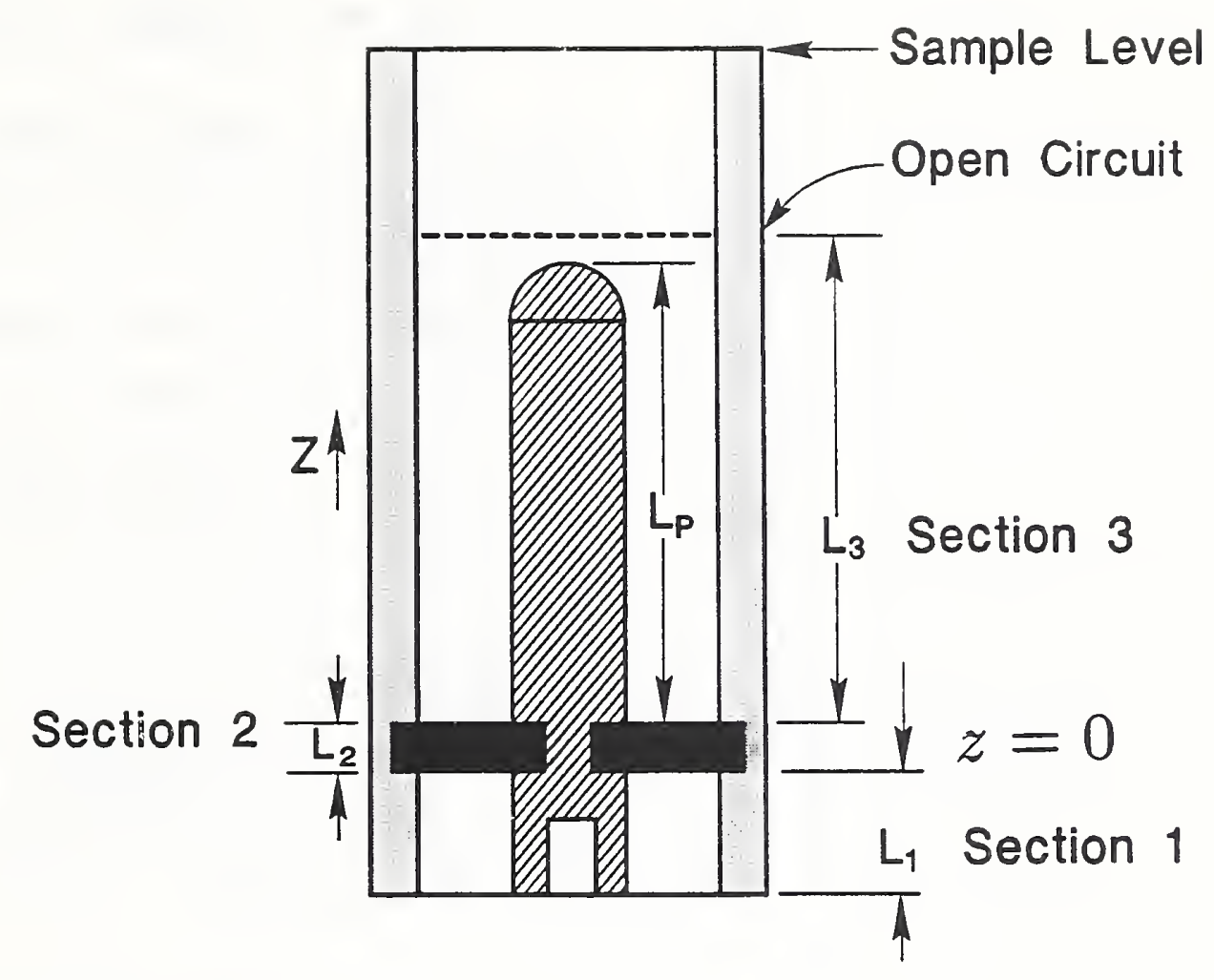

Figure 1.1: The dielectric sample holder with grooves in bead section.

\section{Background}

The shielded open-circuited coaxial line sample holder (OCL) has been used for years for dielectric measurements of liquids and powders [1]. The sample holder operation is based on an accurate model of a coaxial line terminated in a shielded open circuit. The advantage of an open-circuited holder is the ease of sample installation, the broad frequency capability, and the strong electric field in the sample region. The OCL is composed of an outer conductor that extends beyond the end of the inner conductor as shown in figure 1.1. Bussey [1] developed an approximate theoretical model for the shielded open circuit based on the analysis of Somlo [2].

The OCL consists of three sections. Section 1 is the air line section between the bead and the connector, section 2 is the bead, and section 3 contains the sample under test. There are two types of open-circuited holders in common use. The first and most common type of sample holder has machined grooves in the inner and outer conductors in the bead 


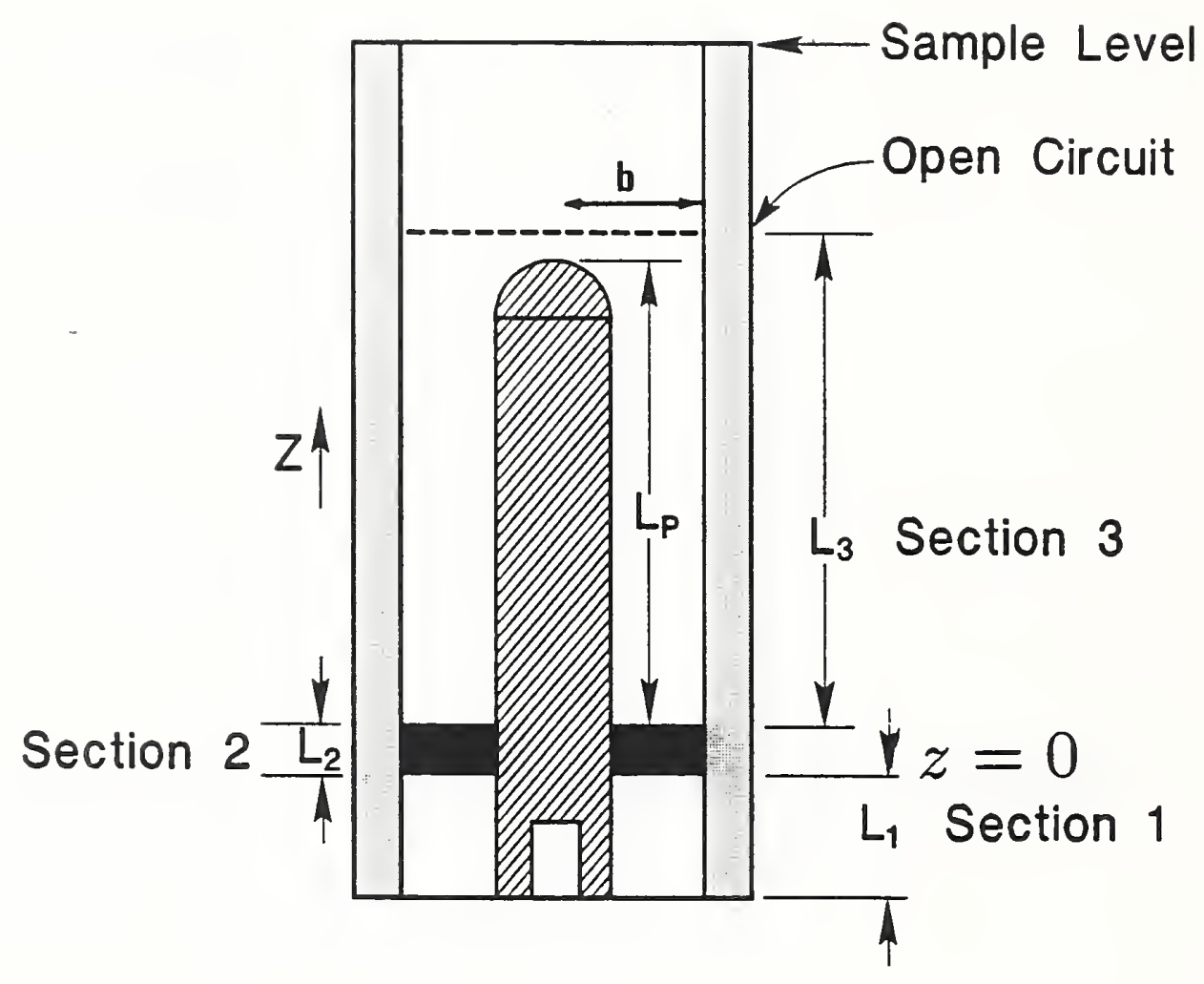

Figure 1.2: The dielectric sample holder for an uncompensated bead.

section. This holder is depicted in figure 1.1. The function of the grooves is to maintain line impedance. Therefore this type of sample holder is called a compensated OCL. For the compensated bead, the fringe capacitance caused by the grooves in the conductors in section 2 can introduce an uncertainty into the effective length of the bead section. The fringing capacitance at the end of the inner conductor also makes the effective length of section 3 slightly longer than the physical length of the inner conductor. Somlo [2] has presented length correction formulas for the shielded open circuit that account for fringing capacitance effects. The second type of OCL is uncompensated and has uncut conductors as depicted in figure 1.2. In this type of holder the impedance varies from section to section.

The literature pertaining to the shielded open circuit is extensive and no attempt is made here to review it exhaustively. von Hippel [3] used an open-circuited sample holder for liquid measurements. Bussey pioneered the development of the OCL discussed in this report [1]. Scott [4] studied the instabilities encountered in solving the relevant nonlinear 
open-circuit equations. Hill [5] studied in situ measurements of soils using open-circuited transmission lines. Jesch [6] used the shielded open-circuited holder for measurements on shale oil. Biological tissues have been measured using the shielded open-circuited line, for example by Stuchly and Stuchly [7]. The sample holder is useful in high-temperature measurements $[6,8]$.

Bussey's formulation of the open-circuited holder [1] is based on an admittance transformation through the various regions of the holder. In this report we express the problem in terms of dominant mode scattering. We develop a compact expression for the reflection coefficient in terms of bead and sample parameters and thereby eliminate the need to transform through the various sections of the sample holder. We extend the formulation to include magnetic measurements and present a differential uncertainty analysis.

\section{Theoretical Formulation}

We begin with a simple mathematical analysis of the electromagnetic fields in the sample holder. The problem is to relate the reflection coefficient to the complex permittivity and permeability.

Consider a sample in a transmission line as indicated in figure 1.1. The effective lengths of the air section, bead section, and sample sections are $L_{1}, L_{2}$, and $L_{3}$. Assuming only the dominant $T E M$ mode (see Appendix) and a $\exp (j \omega t)$ time dependence in the coaxial line, we can write down the following expressions for the normalized, radially integrated electric fields in these three sections

$$
\begin{gathered}
E_{1}(z)=\exp \left(-\gamma_{1} z\right)+\Gamma \exp \left(\gamma_{1} z\right), \\
E_{2}(z)=C_{1} \exp \left(-\gamma_{2} z\right)+C_{2} \exp \left(\gamma_{2} z\right),
\end{gathered}
$$




$$
E_{3}(z)=C_{3} \exp \left(-\gamma_{3}\left(z-L_{2}\right)\right)+C_{4} \exp \left(\gamma_{3}\left(z-L_{2}\right)\right)
$$

where $\Gamma$ is the $T E M$ reflection coefficient and $C_{i}, i=1,2,3,4$ are constants to be determined. The tangential normalized magnetic fields can be found from the electric fields by use of Maxwell's equations

$$
\begin{gathered}
H_{1}(z)=\frac{1}{Z_{1}}\left[\exp \left(-\gamma_{1} z\right)-\Gamma \exp \left(\gamma_{1} z\right)\right], \\
H_{2}(z)=\frac{1}{Z_{2}}\left[C_{1} \exp \left(-\gamma_{2} z\right)-C_{2} \exp \left(\gamma_{2} z\right)\right], \\
H_{3}(z)=\frac{1}{Z_{3}}\left[C_{3} \exp \left(-\gamma_{3}\left(z-L_{2}\right)\right)-C_{4} \exp \left(\gamma_{3}\left(z-L_{2}\right)\right)\right],
\end{gathered}
$$

where $Z_{i}$ are the characteristic wave impedances in the various sections of the line.

The propagation constants of the line sections are

$$
\gamma_{i}=j \frac{\omega}{c_{v a c}} \sqrt{\epsilon_{R(i)}^{*} \mu_{R(i)}^{*}}
$$

and the permittivity and permeability are written as

$$
\epsilon_{i}=\left[\epsilon_{R(i)}^{\prime}-j \epsilon_{R(i)}^{\prime \prime}\right] \epsilon_{o}=\epsilon_{R(i)}^{*} \epsilon_{o}
$$

and

$$
\mu_{i}=\left[\mu_{R(i)}^{\prime}-j \mu_{R(i)}^{\prime \prime}\right] \mu_{o}=\mu_{R(i)}^{*} \mu_{o} .
$$

Here $c_{v a c}$ is the speed of light in vacuum, $\omega$ is the angular frequency, $\epsilon_{o}$ and $\mu_{o}$ are the permittivity and permeability of vacuum, $\epsilon_{R(i)}^{*}$ and $\mu_{R(i)}^{*}$ are the complex relative permittivity and permeability measured in section $(i)$ of the coaxial sample holder.

The model of the open circuit used in this theory is approximate and requires length corrections to be made to the physically measured length of the inner conductor to account 
for fringing capacitance. The first correction is due to the fringing capacitance at the end of the inner conductor. Somlo derived an approximate expression for a correction to the inner conductor length that accounts for the fringing capacitance $[1,2]$

$$
L_{3}^{\prime}=(b-a)\left(0.6034+0.9464 x^{2}+18.19 x^{5.127}\right)
$$

where $x=b \sqrt{\epsilon_{R(3)}^{\prime}} / \lambda, \lambda$ is the free space wavelength, and $b$ and $a$ are the radii of the outer and inner conductors. $L_{3}^{\prime}$ is an increasing function of frequency. Equation (2.10) is valid for $x<0.3$. It is assumed that the frequency of operation is chosen so that the $T M_{01}$ mode is below cut-off in the cylindrical waveguide section. This requires $x<0.383$ or $f_{(G H z)}<0.115 / b \sqrt{\epsilon_{R(3)}^{\prime}}[1]$. The second correction is due to the hemispherical end of the inner conductor. If $L_{p}$ is the measured length of the inner conductor in section 3 from the tip to the end, then the length correction due to the effects of the hemispherical end for a $50 \Omega$ line is [1] $L_{I}=L_{p}-0.42 a$ and for a $75 \Omega$ line is $L_{I}=L_{p}-0.36 a$. Therefore the total effective length of the inner conductor is

$$
L_{3}=L_{I}+L_{3}^{\prime}
$$

For a flat end on the inner conductor $L_{I}=L_{p}$, so $L_{3}=L_{p}+L_{3}^{\prime}$.

We wish to determine the coefficients in eqs (2.1) through (2.3) by imposing boundary conditions on the system of equations. The boundary conditions are:

- the tangential components of the electric and magnetic fields $\left(E_{\rho}, H_{\phi}\right)$ are continuous at sample interfaces $\left(z=0, z=L_{2}\right)$,

- the tangential magnetic field $H_{\phi}$ approaches 0 , at the effective position of the open circuit, $z=L_{2}+L_{3}$.

This last boundary condition is an approximation. However, the approximation is reasonable. The validity of the theoretical model is limited to applications where the $T M_{0 n}$ 
modes are below cut-off in the cylindrical waveguide formed by the extension of the outer conductor.

Equations for the coefficients in eqs (2.1) through (2.6) can be obtained by application of the boundary conditions. If we apply the boundary conditions to eqs (2.1) through (2.3) and (2.4) through (2.6) we obtain

$$
\begin{gathered}
1+\Gamma=C_{1}+C_{2}, \\
C_{1} \exp \left(-\gamma_{2} L_{2}\right)+C_{2} \exp \left(\gamma_{2} L_{2}\right)=C_{3}+C_{4}, \\
\Gamma-1=\beta_{1}\left[C_{2}-C_{1}\right], \\
\beta_{2}\left[C_{1} \exp \left(-\gamma_{2} L_{3}\right)-C_{2} \exp \left(\gamma_{2} L_{3}\right)\right]=C_{3}-C_{4}, \\
C_{4}=\delta C_{3},
\end{gathered}
$$

where

$$
\delta=\exp \left(-2 \gamma_{3} L_{3}\right)
$$

We assume that for air and bead sections $\mu_{R(i)}^{*}=1$. Also

$$
\begin{aligned}
& \beta_{1}=\frac{Z_{1}}{Z_{2}}, \\
& \beta_{2}=\frac{Z_{3}}{Z_{2}} .
\end{aligned}
$$

For a compensated bead system $\beta_{1}=1$ and $\beta_{2}$ reduces to

$$
\beta_{2}=\sqrt{\frac{\mu_{R(3)}^{*}}{\epsilon_{R(3)}^{*}}} .
$$


The boundary-condition equations can be written compactly as

$$
\left(\begin{array}{cccr}
1 & -1 & -1 & 0 \\
0 & \frac{1}{\eta} & \eta & -(1+\delta) \\
1 & \beta_{1} & -\beta_{1} & 0 \\
0 & -\frac{\beta_{2}}{\eta} & \beta_{2} \eta & (1-\delta)
\end{array}\right)\left(\begin{array}{c}
\Gamma \\
C_{2} \\
C_{3} \\
C_{4}
\end{array}\right)=\left(\begin{array}{c}
-1 \\
0 \\
1 \\
0
\end{array}\right),
$$

where

$$
\eta=\exp \left(\gamma_{2} L_{2}\right)
$$

Solution of eq (2.21) yields for the reflection coefficient an equation that is transcendental in the permittivity and permeability:

$$
\Gamma=\exp \left(-2 \gamma_{1} L_{1}\right) \frac{\left(\tanh \gamma_{3} L_{3} \tanh \gamma_{2} L_{2}+\beta_{2}\right)-\beta_{1}\left(\tanh \gamma_{3} L_{3}+\beta_{2} \tanh \gamma_{2} L_{2}\right)}{\left(\tanh \gamma_{3} L_{3} \tanh \gamma_{2} L_{2}+\beta_{2}\right)+\beta_{1}\left(\tanh \gamma_{3} L_{3}+\beta_{2} \tanh \gamma_{2} L_{2}\right)} .
$$

Included in eq (2.23) is an electrical length correction for section 1.

For the special case of a compensated bead, eq (2.23) reduces to

$$
\Gamma=\exp \left(-2\left(\gamma_{1} L_{1}+\gamma_{2} L_{2}\right)\right) \frac{\beta_{2}-\tanh \left(\gamma_{3} L_{3}\right)}{\beta_{2}+\tanh \left(\gamma_{3} L_{3}\right)} .
$$

The dependence on $L_{1}$ can be eliminated by dividing the reflection coefficients of the sample filled holder in eq $(2.23)$ by the reflection coefficients measured on an air-filled sample holder

$$
\Gamma_{a i r}=\exp \left(-2 \gamma_{1}\left(L_{1}+L_{2}+L_{3}\right)\right)
$$

to obtain

$$
\Gamma=\Gamma_{a i r} \exp \left(2 \gamma_{1} L_{3}\right) \frac{\beta_{2}-\tanh \left(\gamma_{3} L_{3}\right)}{\beta_{2}+\tanh \left(\gamma_{3} L_{3}\right)}
$$

\subsection{Numerical Results}

Figure 2.1 shows the magnitude of the reflection coefficient as a function of normalized length. The minima of the reflection coefficient for low-loss samples occurs at half-integral wavelengths in the material. 


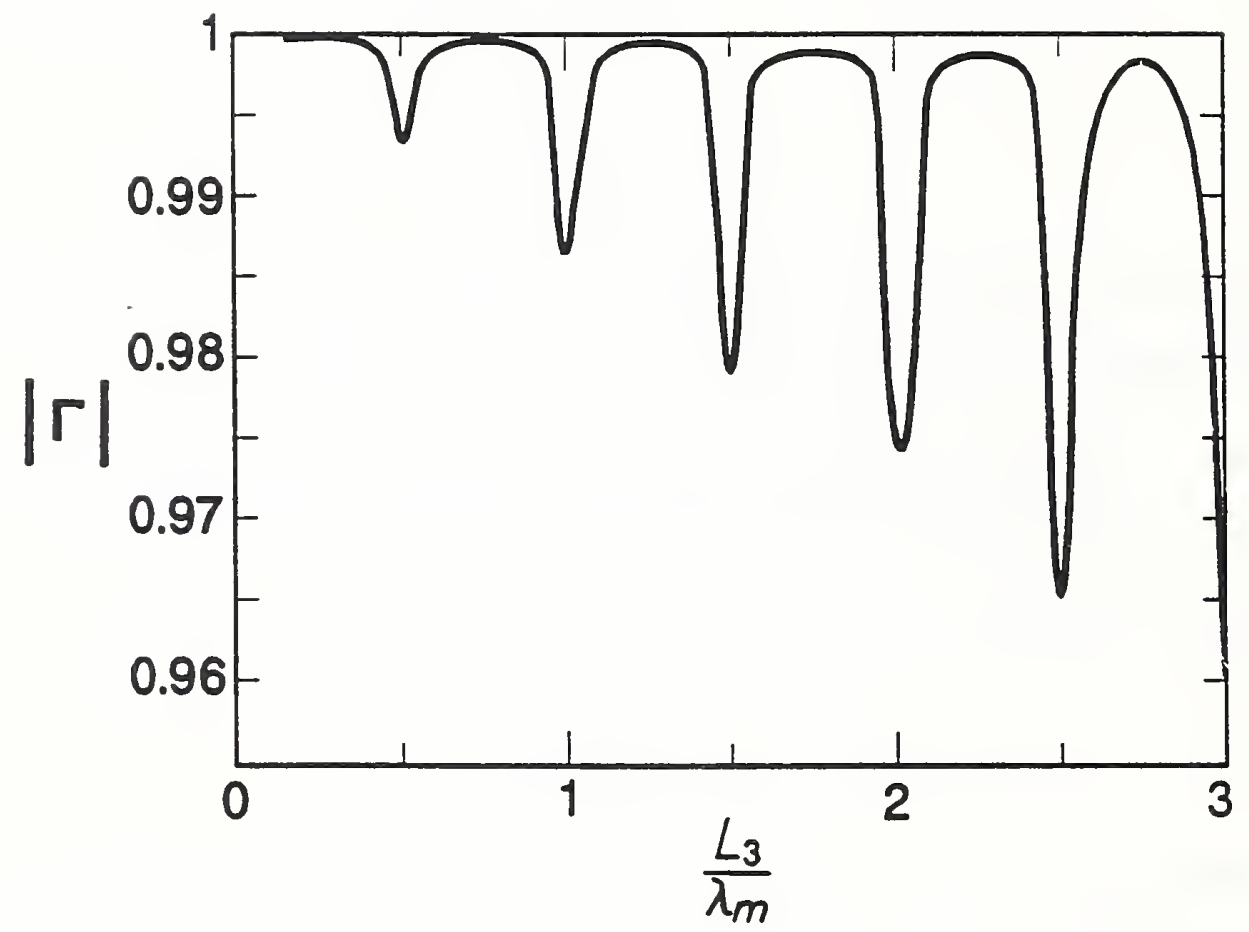

Figure 2.1: The magnitude of the reflection coefficient as a function of normalized length with $\epsilon_{R(3)}^{*}=(20,-0.01)$. 


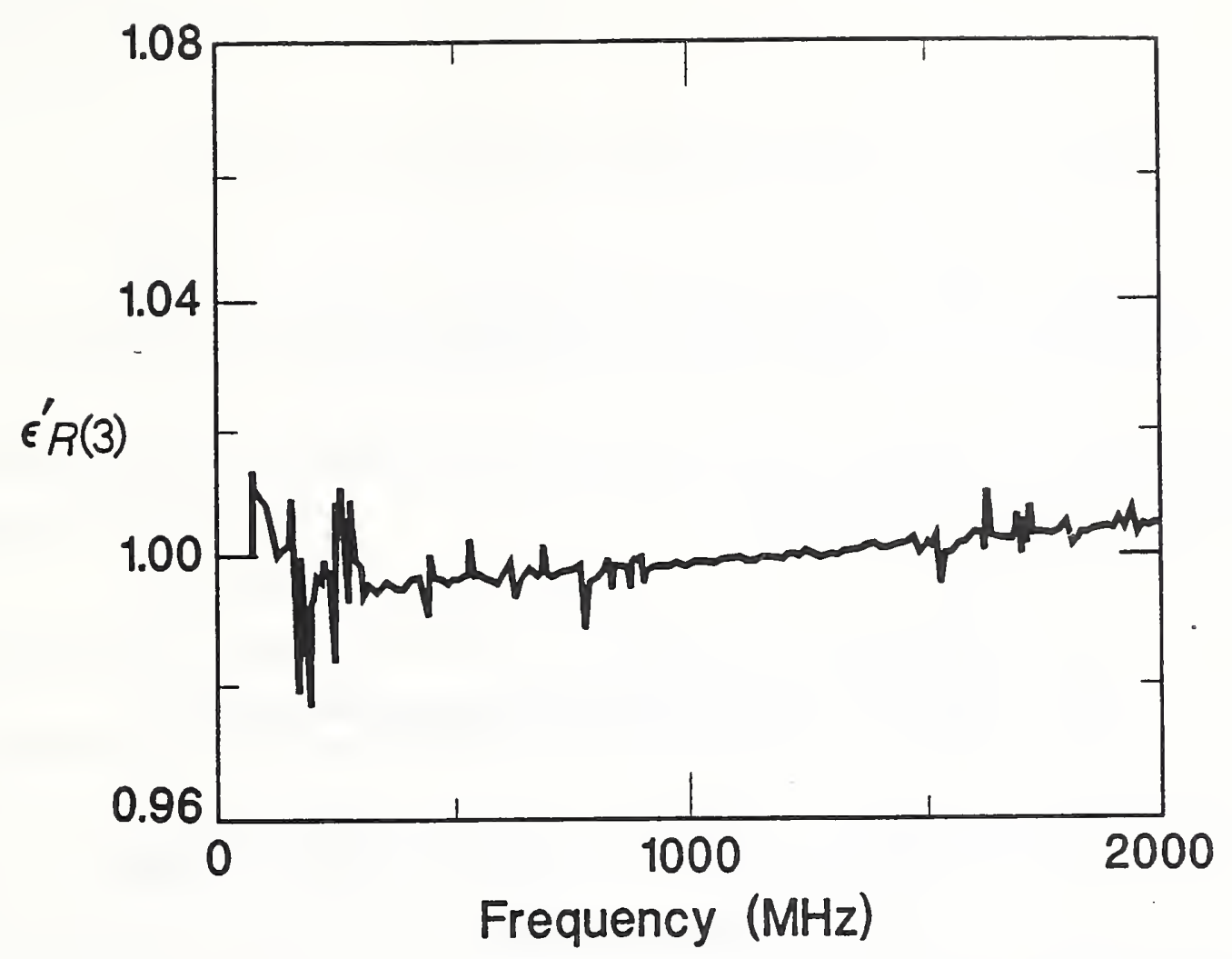

Figure 2.2: The real part of the permittivity of air as a function of frequency (maximum rms uncertainty over the band is $\left.\Delta \epsilon_{R(3)}^{\prime} \pm 0.03\right)$.

The determination of the permittivity proceeds by solving the transcendental equation eq (2.23) which involves the sample length, sample position, and reflection coefficient. With modern computer systems, iterative procedures are easy to implement. In figures 2.2 through 2.4 the calculated permittivities of air and carbonaceous sand as functions of the volume fraction of water are displayed. The uncertainties are due to sample length and scattering parameters. In figure 2.2 we see increased uncertainty at lower frequencies. This is due to the increased relative uncertainty in the phase measurement.

\subsection{Magnetic Measurements}

Two independent measurements are necessary for simultaneous permittivity and permeability determination. This is accomplished for the open-circuited sample holder by taking measurements with two different inner conductor lengths. The measurement with the second inner conductor of length $L_{3(2)}$ can be written as 


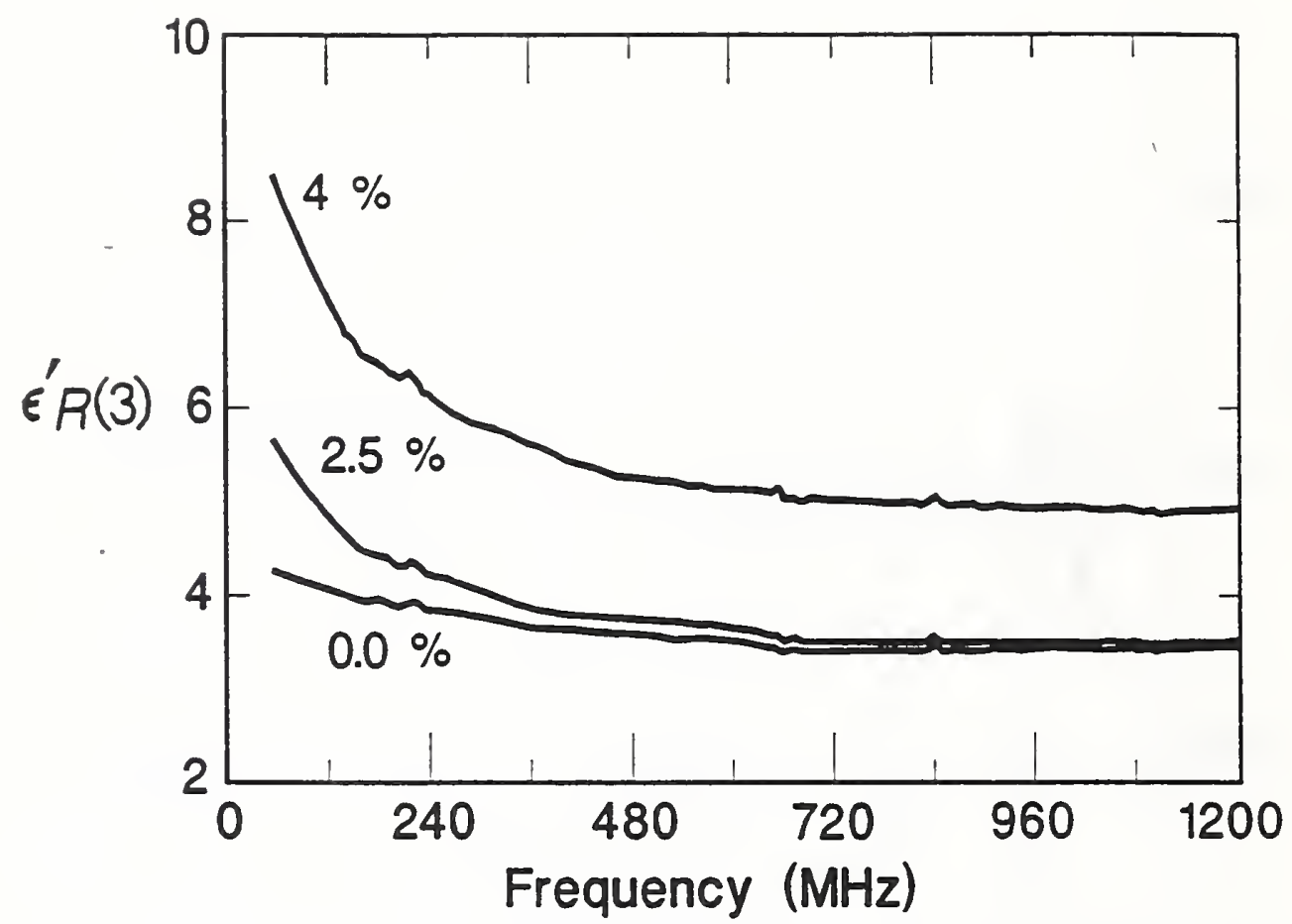

Figure 2.3: The real part of the permittivity of carbonaceous sand mixture as a function of frequency and water volume fraction (maximum rms uncertainty $\Delta \epsilon_{R(3)}^{\prime} \pm 0.35$ ).

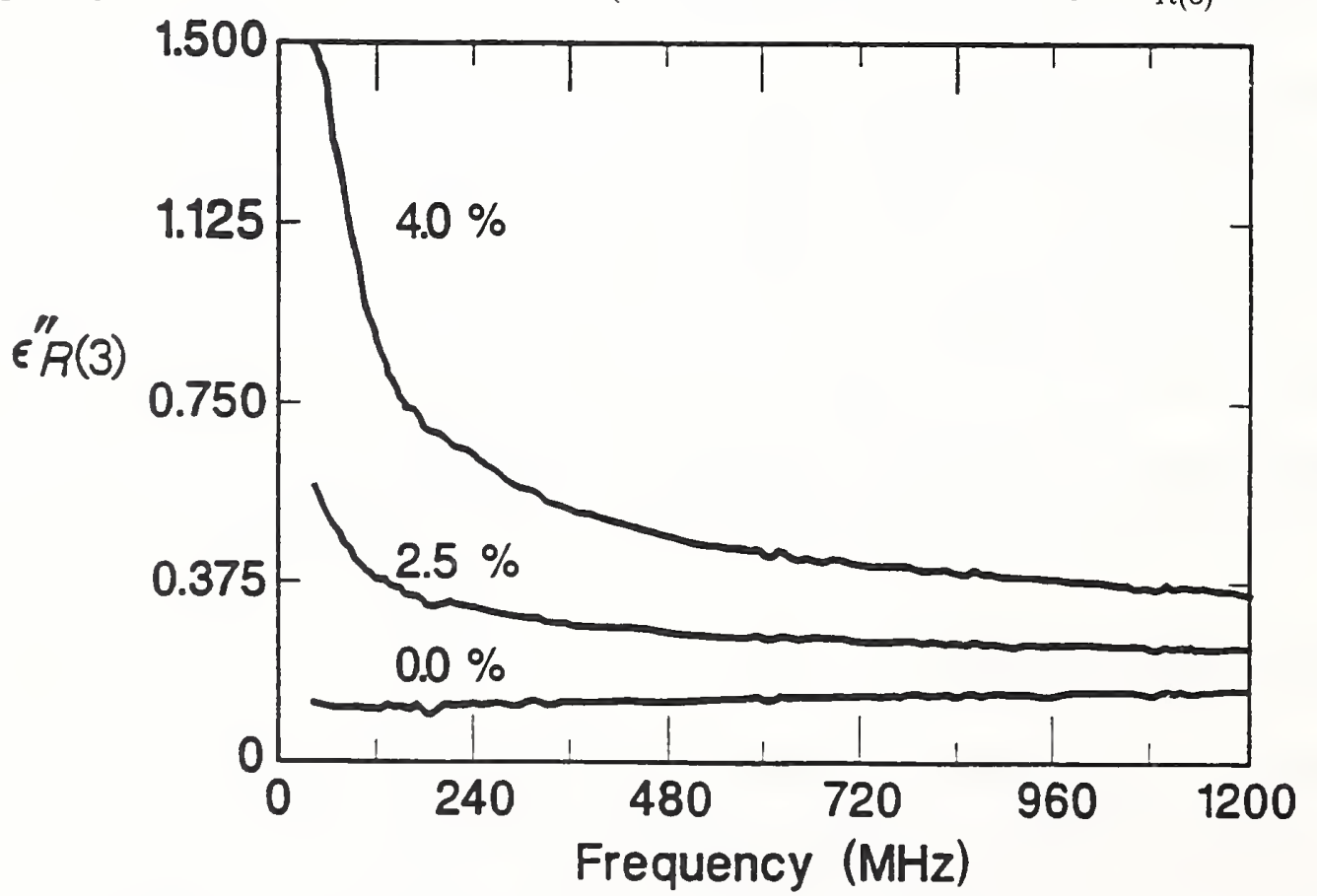

Figure 2.4: The loss of carbonaceous sand mixture as a function of frequency and water volume fraction (maximum rms uncertainty $\Delta \epsilon_{R}^{\prime \prime} \pm 0.15$ ). 


$$
\Gamma_{(2)}=\exp \left(-2 \gamma_{1} L_{1}\right) \frac{\left(\tanh \gamma_{3} L_{3(2)} \tanh \gamma_{2} L_{2}+\beta_{2}\right)-\beta_{1}\left(\tanh \gamma_{3} L_{3(2)}+\beta_{2} \tanh \gamma_{2} L_{2}\right)}{\left(\tanh \gamma_{3} L_{3(2)} \tanh \gamma_{2} L_{2}+\beta_{2}\right)+\beta_{1}\left(\tanh \gamma_{3} L_{3(2)}+\beta_{2} \tanh \gamma_{2} L_{2}\right)}
$$

Equations (2.23) and (2.27) can be solved simultaneously by a Newton-Raphson technique to obtain $\epsilon_{R(3)}^{*}$ and $\mu_{R(3)}^{*}$.

\subsection{Characterization of the Sample Holder}

The thickness and the permittivity of the support bead are important quantities and need to be known very accurately. Since the bead faces are usually not flat, it is difficult to obtain an accurate effective bead thickness. The sample holder bead can be characterized by measuring air in the holder and setting the value to the permittivity of air, which is $\epsilon_{R(1)}^{*}=1.00055$ for an elevation of 1700 meters, and then solving eq (2.23) for the propagation constant of the bead $\left(\gamma_{2}\right)$. Note that in the case of a compensated bead the expression contains only the nondimensional quantity $\gamma_{2} L_{2}$. The effective length of the inner conductor in section 3 can also be studied with this procedure.

\section{Uncertainty Analysis}

Error sources included in the following analysis of the OCL measurement include uncertainties in reflection coefficient, sample dimensions, and bead parameter dimensions. Since this sample holder is usually used for measurements of liquids and powders, there are minimal gaps between conductors and samples. Therefore gap corrections are not applicable. In the case of powders, to obtain a statistically valid result, the mean grain size must be significantly smaller than $b-a$. The uncertainties in the network analyzer parameters usually are well documented by the manufacturer [9].

In order to evaluate the uncertainty introduced by the measured scattering and length parameters, we assume that a differential uncertainty analysis can accurately describe. the 
measurement errors. We assume that the total relative uncertainty with no cross-correlation can be written as

$$
\begin{aligned}
& \frac{\Delta \epsilon_{R(3)}^{\prime}}{\epsilon_{R(3)}^{\prime}}=\frac{1}{\epsilon_{R(3)}^{\prime}} \sqrt{\left(\frac{\partial \epsilon_{R(3)}^{\prime}}{\partial|\Gamma|} \Delta|\Gamma|\right)^{2}+\left(\frac{\partial \epsilon_{R(3)}^{\prime}}{\partial \theta_{11}} \Delta \theta_{11}\right)^{2}+\left(\frac{\partial \epsilon_{R(3)}^{\prime}}{\partial L_{2}} \Delta L_{2}\right)^{2}+\left(\frac{\partial \epsilon_{R(3)}^{\prime}}{\partial L_{3}} \Delta L_{3}\right)^{2}}, \\
& \frac{\Delta \epsilon_{R(3)}^{\prime \prime}}{\epsilon_{R(3)}^{\prime \prime}}=\frac{1}{\epsilon_{R(3)}^{\prime \prime}} \sqrt{\left(\frac{\partial \epsilon_{R(3)}^{\prime \prime}}{\partial|\Gamma|} \Delta|\Gamma|\right)^{2}+\left(\frac{\partial \epsilon_{R(3)}^{\prime \prime}}{\partial \theta_{11}} \Delta \theta_{11}\right)^{2}+\left(\frac{\partial \epsilon_{R(3)}^{\prime \prime}}{\partial L_{2}} \Delta L_{2}\right)^{2}+\left(\frac{\partial \epsilon_{R(3)}^{\prime \prime}}{\partial L_{3}} \Delta L_{3}\right)^{2}},
\end{aligned}
$$

where $\Delta \theta_{11}$ is the uncertainty in the phase of the reflection coefficient, $\Delta|\Gamma|$ is the uncertainty in the magnitude of the reflection coefficient, and $\Delta L_{i}$ are uncertainties in lengths. The uncertainties used for the scattering parameters depend on the specific automatic network analyzer used for the measurements.

In the analysis that follows it is useful to define the phase parameters

$$
\begin{aligned}
& \theta_{2}=\gamma_{2} L_{2}, \\
& \theta_{3}=\gamma_{3} L_{3} .
\end{aligned}
$$

If we define the following function assuming $L_{1}=0$

$$
f=\frac{\left(\tanh \theta_{3} \tanh \theta_{2}+\beta_{2}\right)-\beta_{1}\left(\tanh \theta_{3}+\beta_{2} \tanh \theta_{2}\right)}{\left(\tanh \theta_{3} \tanh \theta_{2}+\beta_{2}\right)+\beta_{1}\left(\tanh \theta_{3}+\beta_{2} \tanh \theta_{2}\right)}-\Gamma=0,
$$

then we can calculate the various derivatives with respect to the independent variables

$$
\frac{\partial f}{\partial \epsilon_{R(3)}^{*}} \frac{\partial \epsilon_{R(3)}^{*}}{\partial L_{2}}+\frac{\partial f}{\partial L_{2}}=0
$$

or

$$
\frac{\partial \epsilon_{R(3)}^{*}}{\partial L_{2}}=-\frac{\frac{\partial f}{\partial L_{2}}}{\frac{\partial f}{\partial \epsilon_{R(3)}^{f}}},
$$


and

$$
\frac{\partial f}{\partial \epsilon_{R(3)}^{*}} \frac{\partial \epsilon_{R(3)}^{*}}{\partial L_{3}}+\frac{\partial f}{\partial L_{3}}=0
$$

or

$$
\frac{\partial \epsilon_{R(3)}^{*}}{\partial L_{3}}=-\frac{\frac{\partial f}{\partial L_{3}}}{\frac{\partial f}{\partial \epsilon_{R(3)}^{*}}}
$$

and

$$
\frac{\partial f}{\partial \epsilon_{R(3)}^{*}} \frac{\partial \epsilon_{R(3)}^{*}}{\partial|\Gamma|}+\frac{\partial f}{\partial|\Gamma|}=0
$$

or

$$
\frac{\partial \epsilon_{R(3)}^{*}}{\partial|\Gamma|}=-\frac{\frac{\partial f}{\partial|\Gamma|}}{\frac{\partial f}{\partial \epsilon_{R(3)}^{*}}} .
$$

It is apparent that

$$
\frac{\partial \epsilon_{R(3)}^{*}}{\partial \theta_{11}}=j|\Gamma| \frac{\partial \epsilon_{R(3)}^{*}}{\partial|\Gamma|},
$$

and

$$
\frac{\partial f}{\partial|\Gamma|}=-\exp \left(j \theta_{11}\right) .
$$

The required derivatives can be calculated explicitly

$$
\begin{aligned}
& \frac{\partial f}{\partial L_{2}}= \\
& \gamma_{2} \operatorname{sech}^{2} \theta_{2}\left[\frac{\left(\tanh \theta_{3}-\beta_{1} \beta_{2}\right)}{\left(\tanh \theta_{3} \tanh \theta_{2}+\beta_{2}\right)+\beta_{1}\left(\tanh \theta_{3}+\beta_{2} \tanh \theta_{2}\right)}-\right. \\
& \left.\left(\tanh \theta_{3}+\beta_{1} \beta_{2}\right) \frac{\left(\tanh \theta_{3} \tanh \theta_{2}+\beta_{2}\right)-\beta_{1}\left(\tanh \theta_{3}+\beta_{2} \tanh \theta_{2}\right)}{\left(\left(\tanh \theta_{3} \tanh \theta_{2}+\beta_{2}\right)+\beta_{1}\left(\tanh \theta_{3}+\beta_{2} \tanh \theta_{2}\right)\right)^{2}}\right] \\
& \frac{\partial f}{\partial L_{3}}=\gamma_{3} \operatorname{sech}^{2}\left[\theta_{3} \frac{\left(\tanh \theta_{2}-\beta_{1}\right)}{\left(\tanh \theta_{3} \tanh \theta_{2}+\beta_{2}\right)+\beta_{1}\left(\tanh \theta_{3}+\beta_{2} \tanh \theta_{2}\right)}-\right. \\
& \left.\left(\tanh \theta_{2}+\beta_{1}\right) \frac{\left(\tanh \theta_{3} \tanh \theta_{2}+\beta_{2}\right)-\beta_{1}\left(\tanh \theta_{3}+\beta_{2} \tanh \theta_{2}\right)}{\left(\left(\tanh \theta_{3} \tanh \theta_{2}+\beta_{2}\right)+\beta_{1}\left(\tanh \theta_{3}+\beta_{2} \tanh \theta_{2}\right)\right)^{2}}\right]
\end{aligned}
$$




$$
\begin{aligned}
& \frac{\partial f}{\partial \epsilon_{R(3)}^{*}} . \\
& =\left[\frac{\left(L_{3} \operatorname{sech}^{2} \theta_{3} \tanh \theta_{2} \frac{\partial \gamma_{3}}{\partial \epsilon_{R(3)}^{*}}+\frac{\partial \beta_{2}}{\partial \epsilon_{R(3)}^{*}}\right)-\beta_{1}\left(L_{3} \operatorname{sech}^{2} \theta_{3} \frac{\partial \gamma_{3}}{\partial \epsilon_{R(3)}^{*}}+\frac{\partial \beta_{2}}{\partial \epsilon_{R(3)}^{*}} \tanh \theta_{2}\right)}{\left(\tanh \theta_{3} \tanh \theta_{2}+\beta_{2}\right)+\beta_{1}\left(\tanh \theta_{3}+\beta_{2} \tanh \theta_{2}\right)}\right. \\
& -\frac{\left(L_{3} \operatorname{sech}^{2} \theta_{3} \tanh \theta_{2} \frac{\partial \gamma_{3}}{\partial \epsilon_{R(3)}^{*}}+\frac{\partial \beta_{2}}{\partial \epsilon_{R(3)}^{*}}\right)+\beta_{1}\left(L_{3} \operatorname{sech}^{2} \theta_{3} \frac{\partial \gamma_{3}}{\partial \epsilon_{R(3)}^{*}}+\frac{\partial \beta_{2}}{\partial \epsilon_{R(3)}^{*}} \tanh \theta_{2}\right)}{\left.\left(\tanh \theta_{3} \tanh \theta_{2}+\beta_{2}\right)+\beta_{1}\left(\tanh \theta_{3}+\beta_{2} \tanh \theta_{2}\right)\right)^{2}} \\
& \left.\times\left[\left(\tanh \theta_{3} \tanh \theta_{2}+\beta_{2}\right)-\beta_{1}\left(\tanh \theta_{3}+\beta_{2} \tanh \theta_{2}\right)\right]\right], \\
& \frac{\partial \gamma_{3}}{\partial \epsilon_{R(3)}^{*}}=j \omega \frac{\sqrt{\mu_{0} \epsilon_{0} \mu_{R(3)}^{*}}}{2 \sqrt{\epsilon_{R(3)}^{*}}} \\
& \frac{\partial \beta_{2}}{\partial \epsilon_{R(3)}^{*}}=-\frac{1}{2 \epsilon_{R(3)}^{*} \sqrt{\frac{\mu_{R(3)}^{*}}{\epsilon_{R(3)}^{*}}}} .
\end{aligned}
$$

The components of the various derivatives in eqs (3.9) through (3.12) are plotted in figures 3.1 through 3.4 as a function of normalized length in the material. The wavelength in the material is $\lambda_{m}=\Re\left(2 \pi c_{v a c} / \omega \sqrt{\epsilon_{R(3)}^{*} \mu_{R(3)}^{*}}\right)$.

Equation (3.12) provides a way of obtaining derivatives with respect to magnitude once the phase derivatives are known. For this reason not all derivatives are plotted. In figures 3.5 through 3.10 the uncertainties in $\epsilon_{R(3)}^{\prime}$ and $\mu_{R(3)}^{\prime}$ are plotted as a function of normalized sample length. The minimum uncertainty for low-loss materials occurs at multiples of onehalf wavelength. These minima correlate with the minima in the reflection coefficient in figure 2.1. In figure 3.10 the frequency dependence of the uncertainty is displayed. 


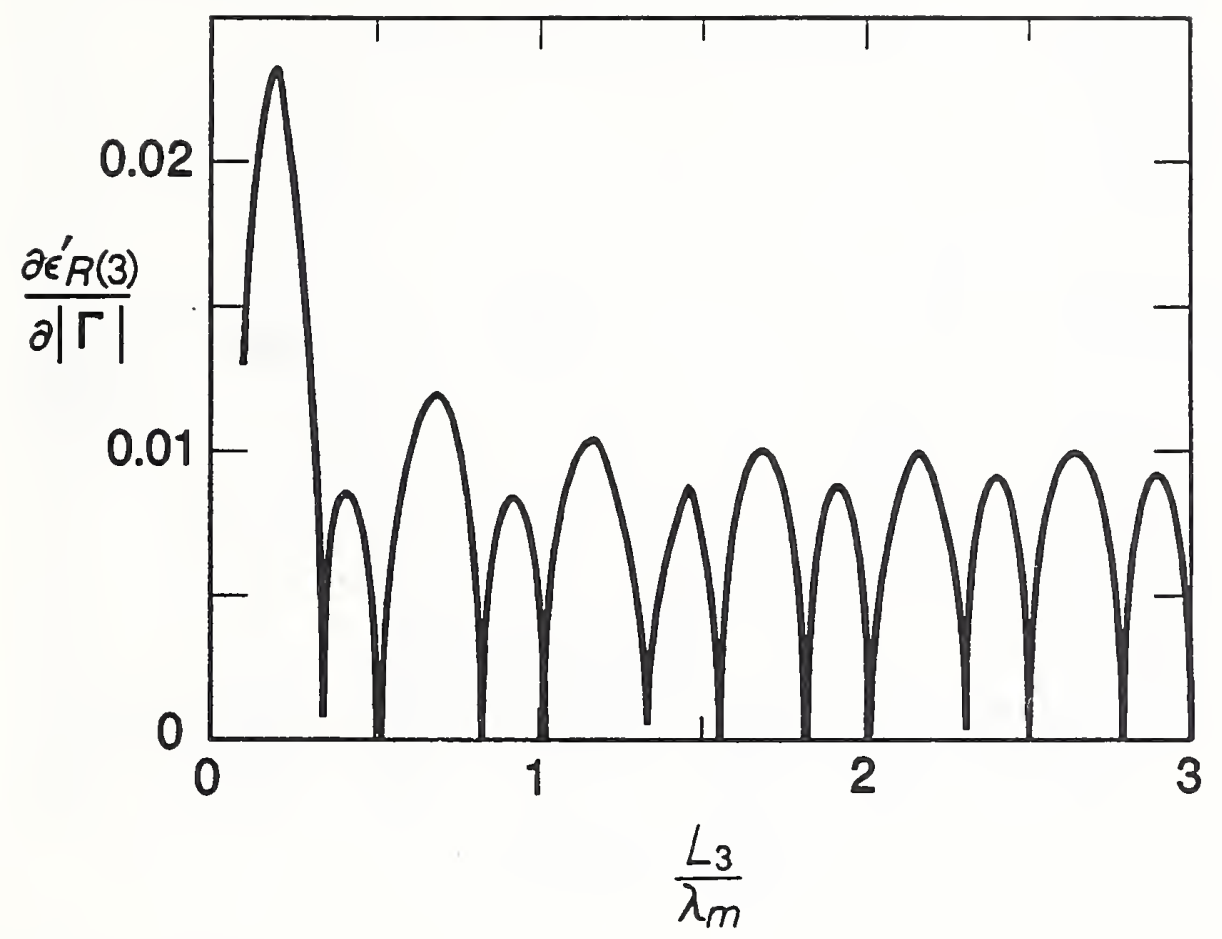

Figure 3.1: The derivative of $\epsilon_{R(3)}^{\prime}$ with respect to $|\Gamma|$ with $\epsilon_{R(3)}^{*}=(5,-0.01)$.

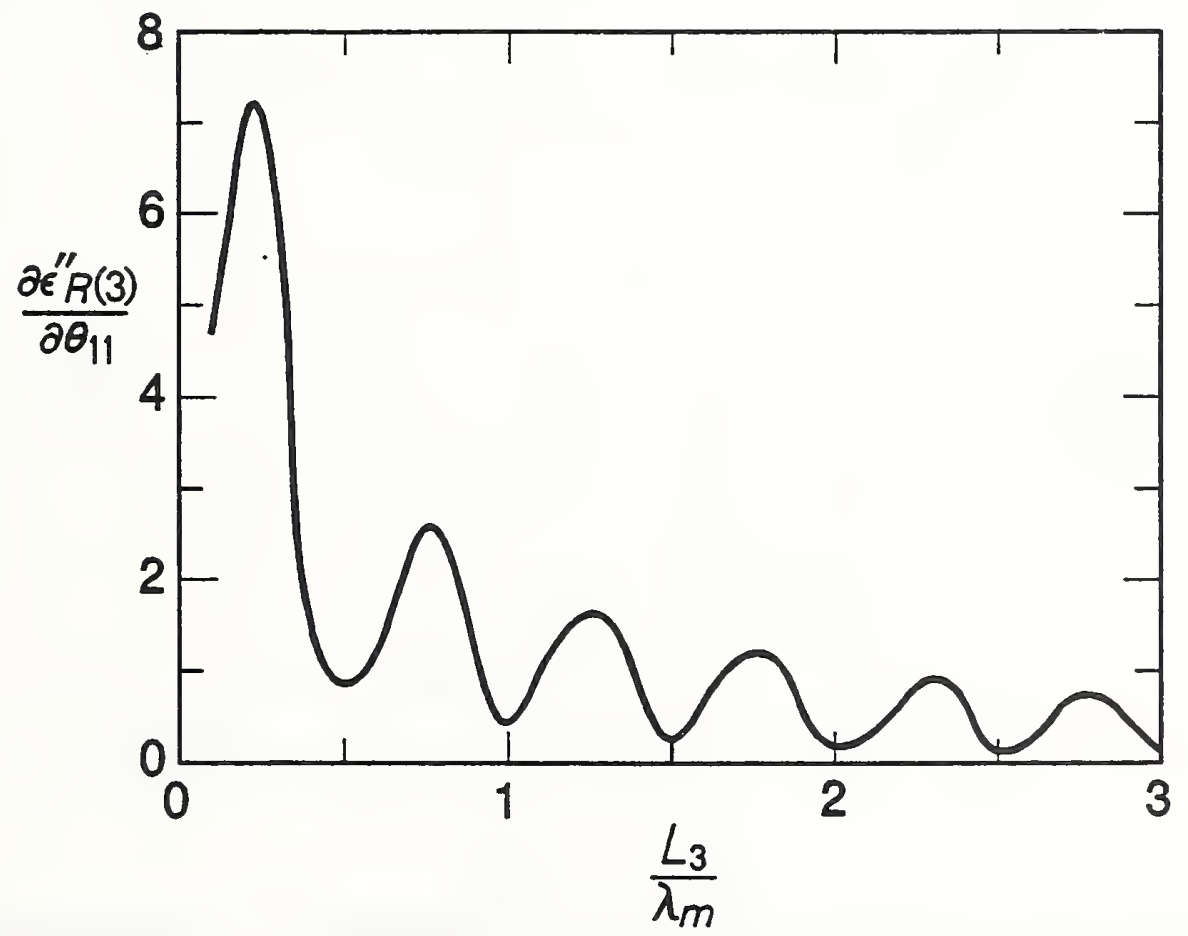

Figure 3.2: The derivative of $\epsilon_{R(3)}^{\prime \prime}$ with respect to $\theta_{11}$ with $\epsilon_{R(3)}^{*}=(5,-0.01)$. 


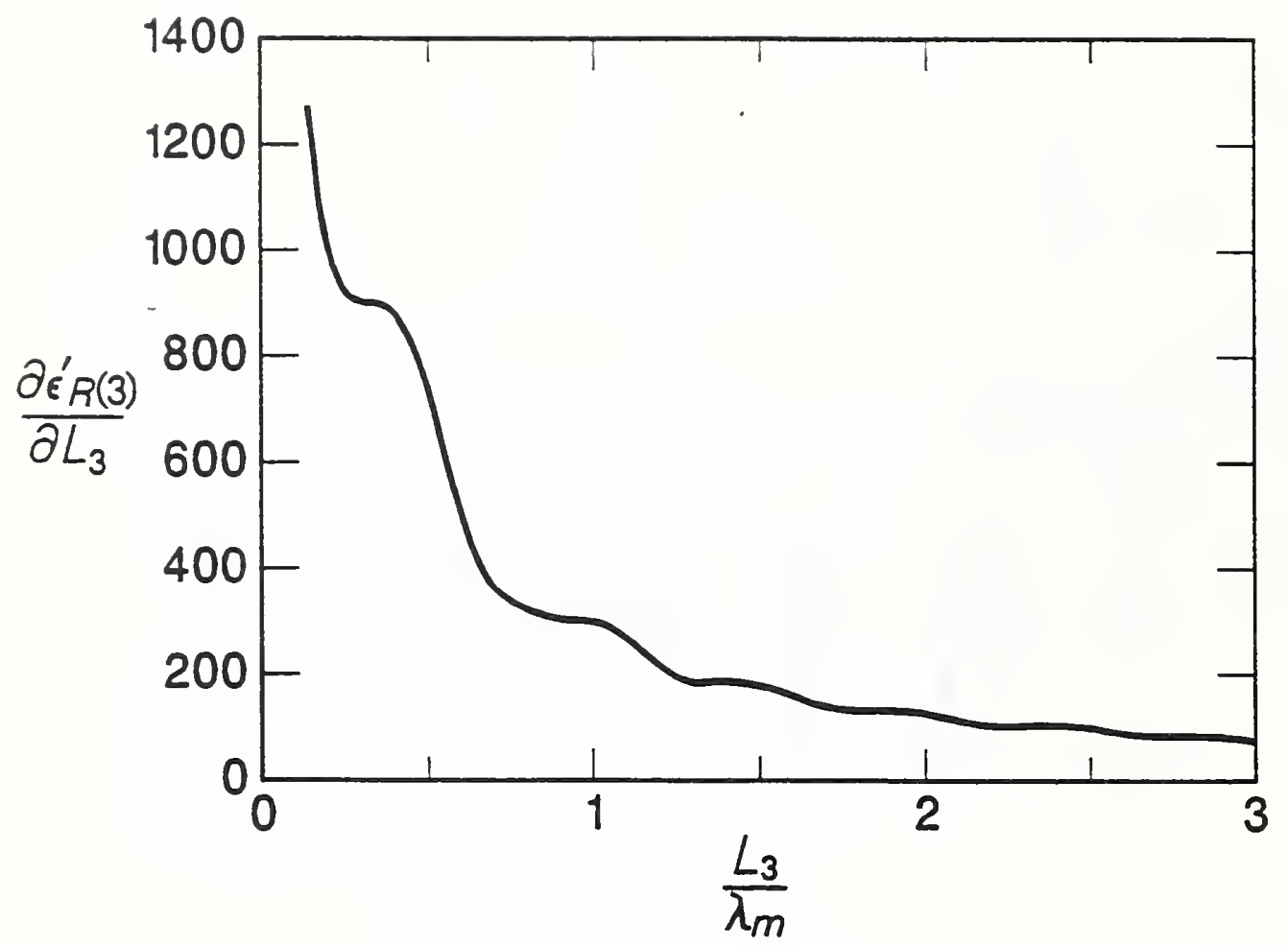

Figure 3.3: The derivative of $\epsilon_{R(3)}^{\prime}$ with respect to normalized length of the inner conductor with $\epsilon_{R(3)}^{*}=(5,-0.01)$.

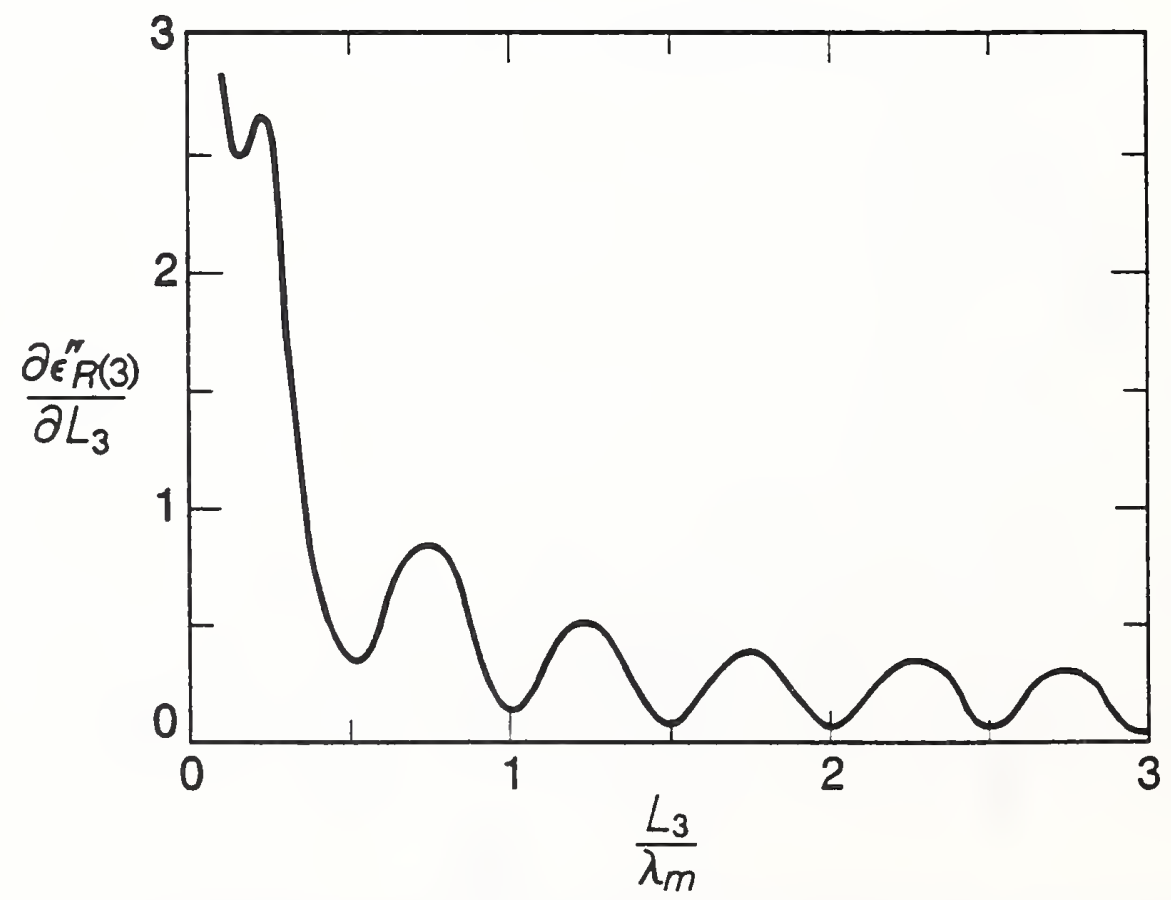

Figure 3.4: The derivative of $\epsilon_{R(3)}^{\prime \prime}$ with respect to normalized length of the inner conductor with $\epsilon_{R(3)}^{*}=(5,-0.01)$. 


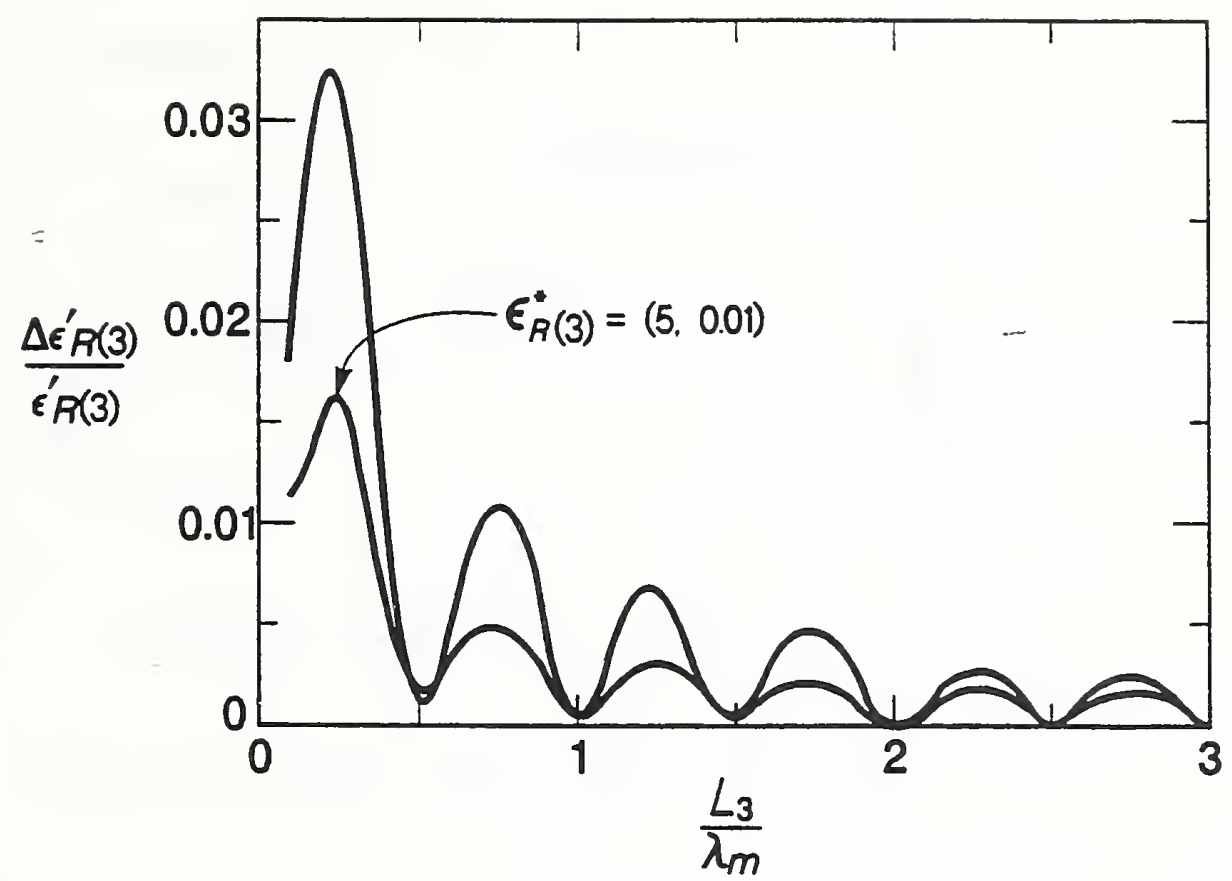

Figure 3.5: The relative uncertainty in $\epsilon_{R(3)}^{\prime}$ for a low-loss material as a function of normalized length with $\epsilon_{R(3)}^{*}=(5,-0.01)$ and $(20,-0.01)$.

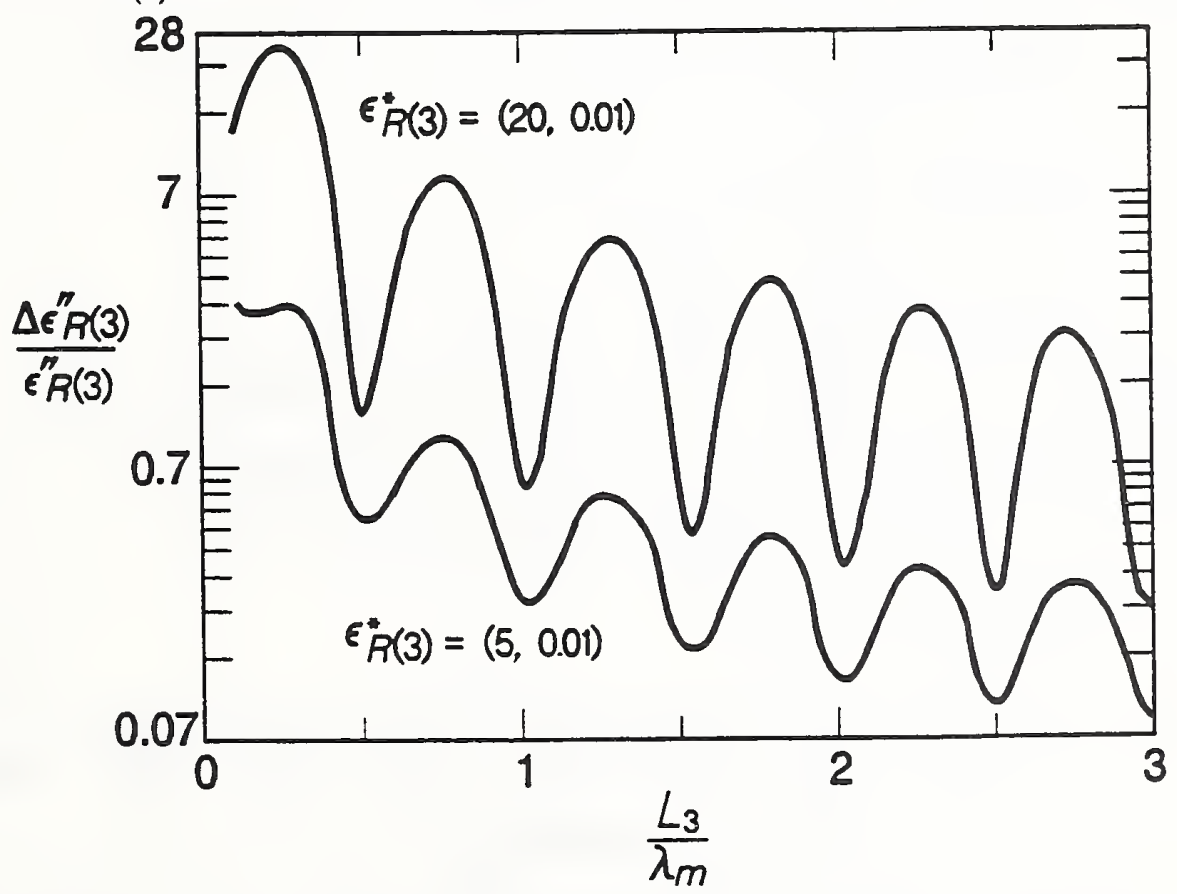

Figure 3.6: The relative uncertainty in $\epsilon_{R(3)}^{\prime \prime}$ for a low-loss material as a function of normalized length with $\epsilon_{R(3)}^{*}=(5,-0.01)$ and $(20,-0.01)$. 


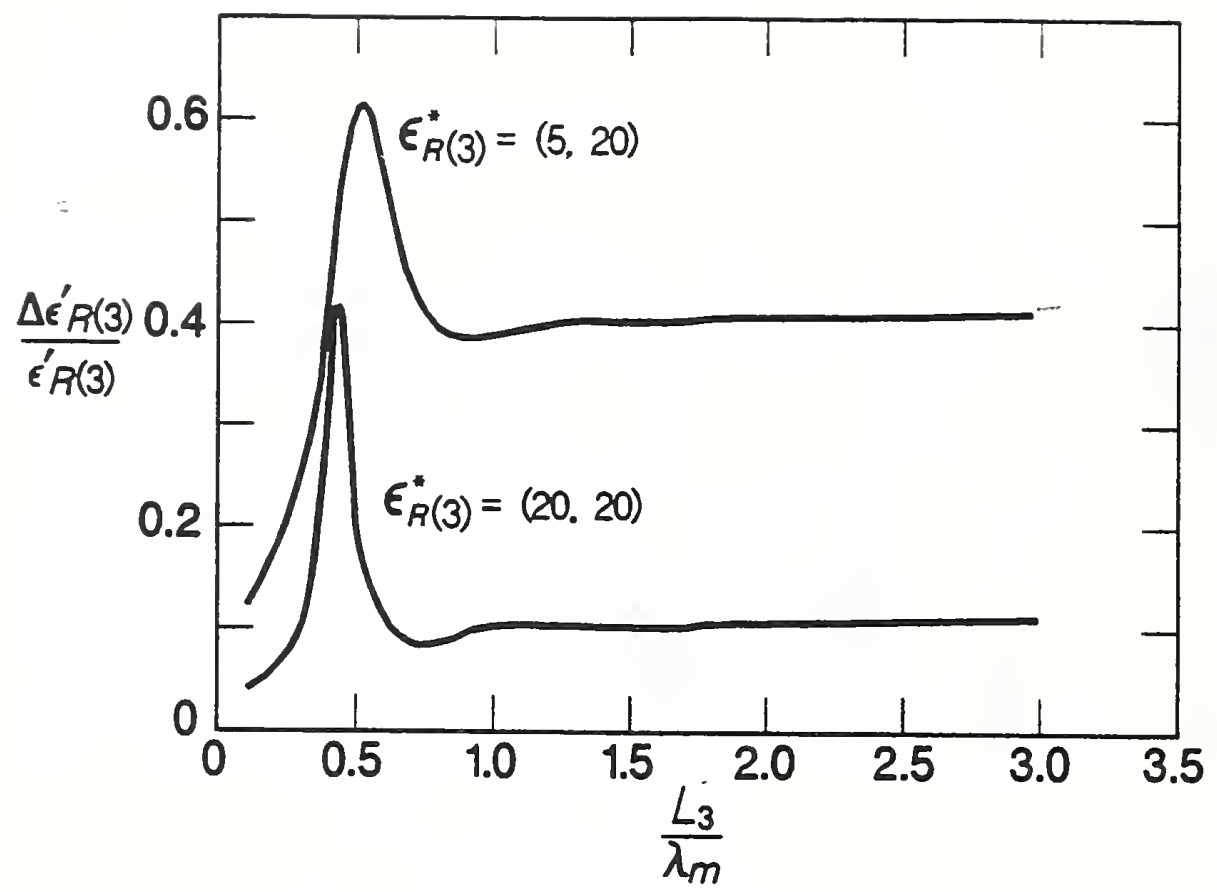

Figure 3.7: The relative uncertainty in $\epsilon_{R(3)}^{\prime}$ for a high-loss material as a function of normalized length with $\epsilon_{R(3)}^{*}=(5,-10)$ and $(20,-20)$.

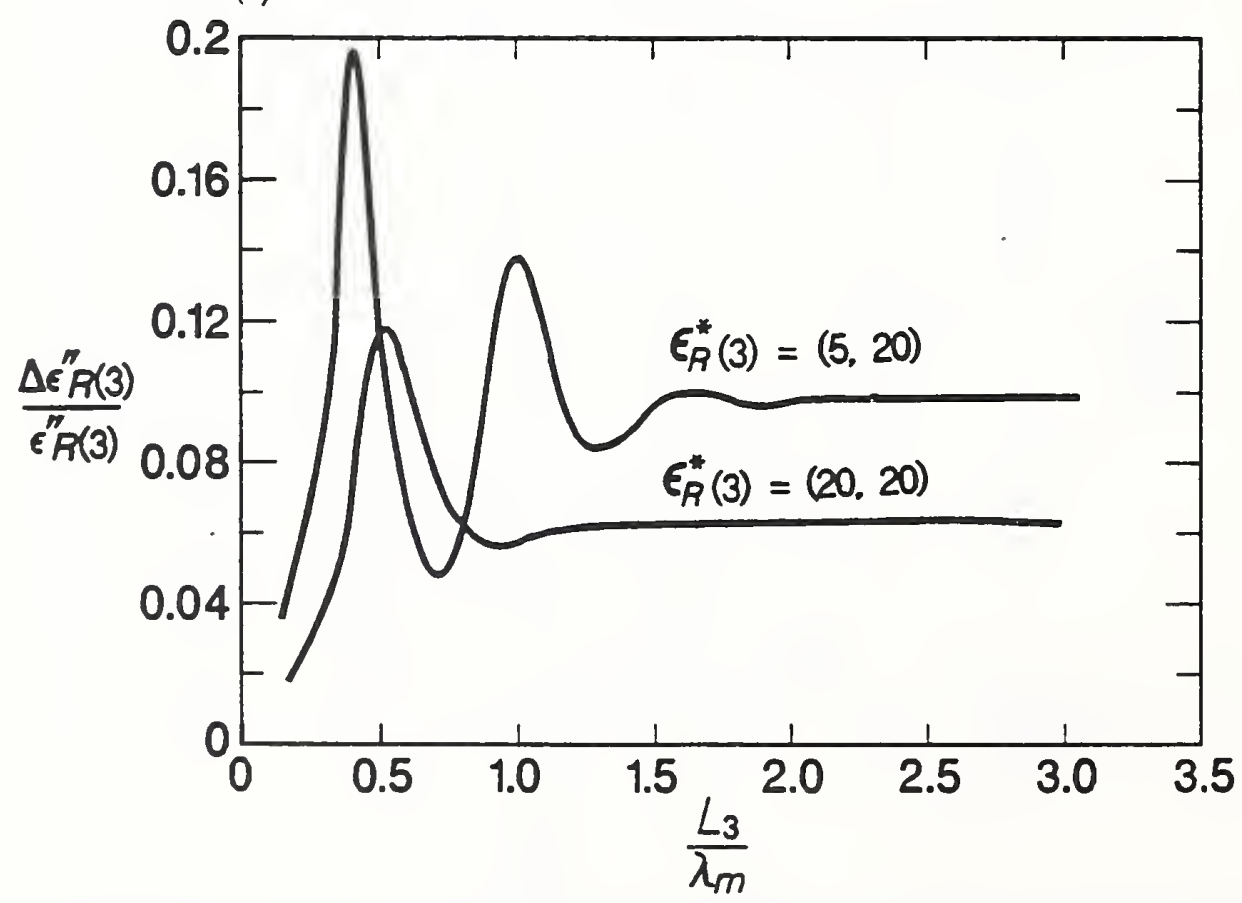

Figure 3.8: The relative uncertainty in $\epsilon_{R(3)}^{\prime \prime}$ for a high-loss material as a function of normalized length with $\epsilon_{R(3)}^{*}=(5,-10)$ and $(20,-20)$. 


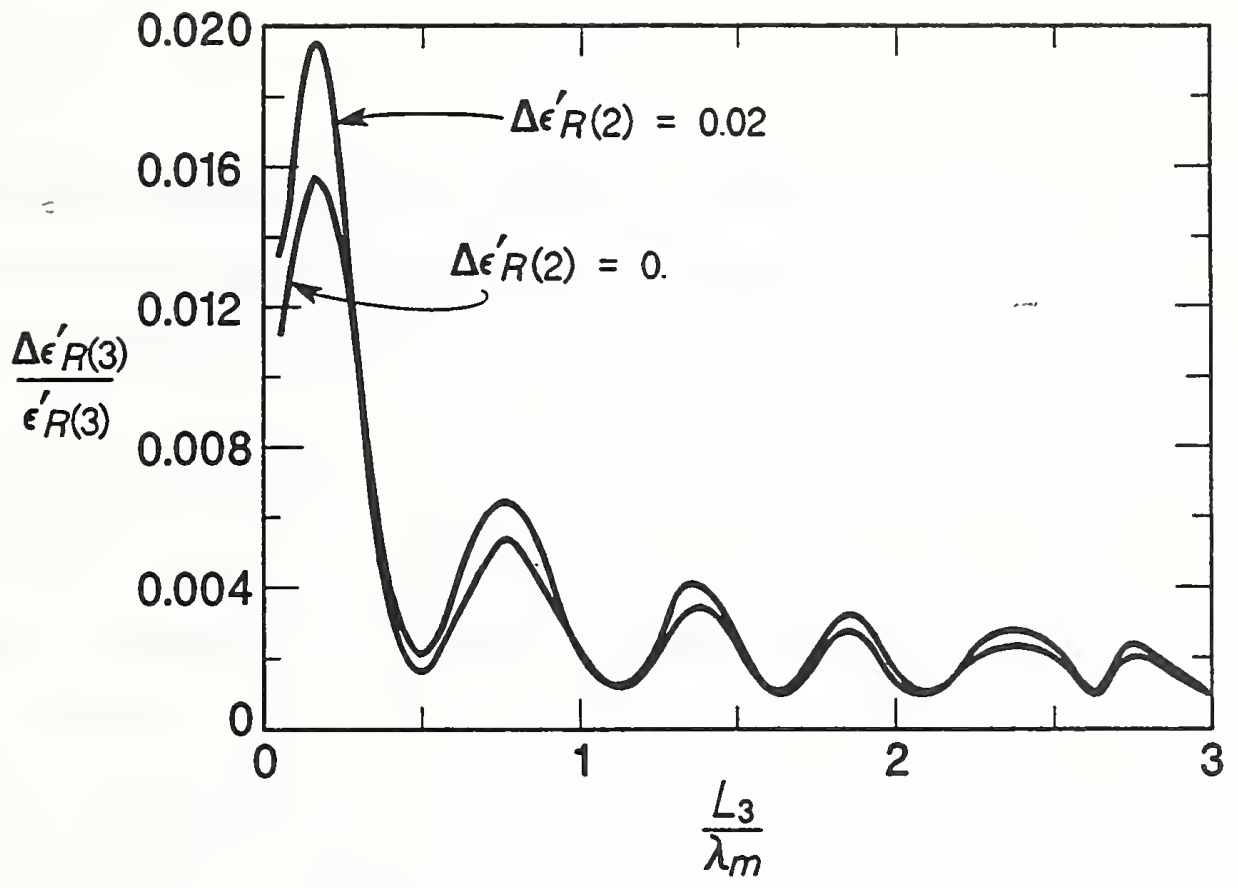

Figure 3.9: The relative uncertainty in $\epsilon_{R(2)}^{\prime}$ for the bead with $\epsilon_{R(2)}^{*}=(2.03,0)$ and for uncertainties in the bead permittivity of $\Delta \epsilon_{R(2)}^{\prime}=0.02$ and $\Delta \epsilon_{R(2)}^{\prime}=0$.

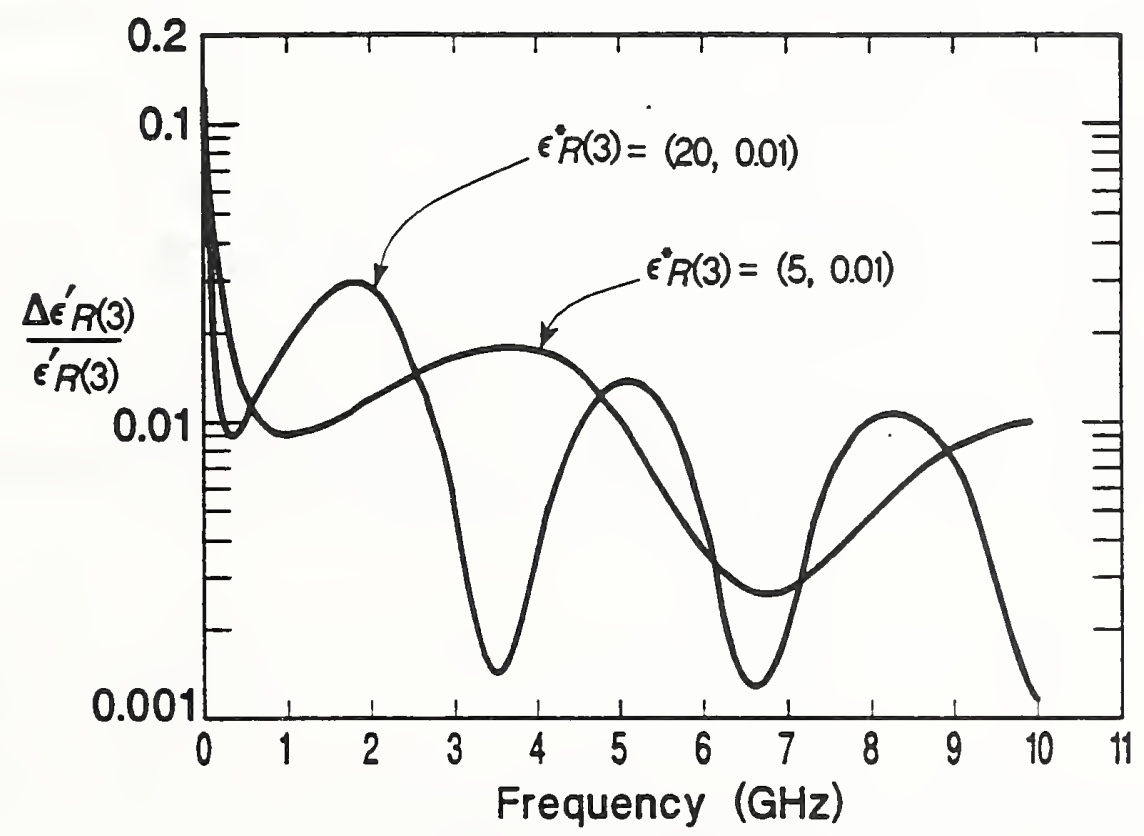

Figure 3.10: The relative uncertainty in $\epsilon_{R(3)}^{\prime}$ as a function of frequency for permittivities $\epsilon_{R(3)}^{*}=(5,-0.01)$ and $\epsilon_{R(3)}^{*}=(20,-0.01)$. 


\section{Discussion}

In coaxial line $\gamma_{2} L$ contains the product of angular frequency, sample length, and the square root of permittivity and permeability. Therefore the effects of sample length and frequency variations are correlated, so plots of various uncertainty parameters as a function of normalized length are particularly informative.

As indicated in figures 3.1 through 3.4 we generally see a decrease in relative uncertainty as a function of increasing sample length and as a function of increasing frequency. The lárge uncertainty at lower frequencies indicates a limitation of the method. This results from the very small phase shift over the length of a sample at low frequencies. At low frequencies the real part of the permittivity is very sensitive to measured phase and sample length. As indicated in figure 2.1, the minima of the reflection coefficient for low-loss materials occur at $n \lambda_{m} / 2$, where $n=1,2,3 \ldots$. For low-loss materials these minima in the reflection coefficient correspond to regions of minimum uncertainty in the real part of the permittivity. Therefore the minimum uncertainty for low-loss materials occurs at frequencies where there is maximal interaction of the fields with the sample. For highloss materials both the real and the imaginary parts of the permittivity are sensitive to the sample lengths. In the measurement of air in figure 2.2 we see that the uncertainty decreases as frequency decreases. This is consistent with the uncertainty analysis results.

The accuracy to which the bead section permittivity is known is important as indicated in figure 3.9. Errors in these values will cause systematic uncertainties in the measurements.

We acknowledge Howard Bussey for various discussions. This work was funded in part by a contract from the Office of Intelligent Processing of Materials. 


\section{References}

[1] Bussey, H. E., "Dielectric measurements in a shielded open circuit coaxial line," IEEE Trans. Instrum. Meas., vol. IM-29, pp. 120-124, June 1980.

[2] Somlo, P. I., "The discontinuity capacitance and the effective position of a shielded open circuit in a coaxial line," Proc. Inst. Radio Elec. Eng. Australia, vol. 28, pp. 7-9, January 1967.

[3] von Hippel, A., Dielectric Materials and Applications. Cambridge, MA: M.I.T. Press, 1954.

[4] Scott, W. R. and Smith, G. S., "Error analysis for dielectric spectroscopy using shielded open-circuited coaxial lines of general length," IEEE Trans. Instrum. Meas., vol. IM-35, pp. 130-137, June 1986.

[5] Hill, P. N., and Green, H. E., "In situ measurement of soil permittivity and permeability," J. Elect. Electron. Eng., Australia, vol. 2, pp. 205-209, December 1982.

[6] Jesch, R. L., "Dielectric measurements of oil shale as functions of temperature and frequency," IEEE Trans. Geosci. Remote Sensing, vol. GE-22, pp. 99-105, March 1984.

[7] Stuchly, M. A. and Stuchly, S. S., "Coaxial line reflection methods for measuring dielectric properties of biological substances at radio and microwave frequencies-A review," IEEE Trans. Instrum. Meas., vol. IM-29, pp. 176-183, 1980.

[8] Baker-Jarvis, J. and Inguva, R., "Dielectric heating of oilshales by monopole and coaxial applicators," J. Micro. Power, vol. 23, pp. 160-170, 1988.

[9] "Measuring dielectric constant with the HP 8510 network analyzer," Product note no. 8510-3, Hewlett-Packard, 1985. 


\section{Appendix: Field Analysis of the Shielded Open Circuit}

In this appendix we will review the electromagnetic fields around the open circuit. It is assumed that materials are homogeneous and isotropic, so there is only an azimuthal component of the magnetic field. The open-circuit position is assumed to be below the fill level of the liquid or powder.

Consider a coaxial line terminated by a shielded open circuit. The frequency of operation is assumed to be such that the modes in the shield region are evanescent. The dominant $T E M$ mode is assumed to be incident upon the open circuit where a reflected TEM wave is formed together with evanesecent $T M_{0 n}$ modes. No energy is lost to the evanescent modes unless the lines are lossy or a lossy material is in the coaxial line or shield.

The assumed existence of only an azimuthal magnetic field requires that the materials are homogeneous and isotropic. Under these assumptions the magnetic field satisfies

$$
\left[\frac{\partial^{2}}{\partial \rho^{2}}+\frac{1}{\rho} \frac{\partial}{\partial \rho}-\frac{1}{\rho^{2}}+\frac{\partial^{2}}{\partial z^{2}}+k_{i}^{2}\right] H_{\phi(i)}(\rho, z)=0,
$$

both in the coaxial line region (3) and the shield region (4).

Since the Laplacian is separable, the solution to the radial electric field in the coaxial line can be written as a linear combination of $T E M$ and $T M_{0 n}$ modes.

In both the coaxial line and shield it is required that at the inner and outer conducting surfaces $E_{z}=0$. The z-component of the electric field is given by

$$
E_{z(i)}=\frac{1}{j \omega \epsilon_{i}}\left[\frac{\partial H_{\phi(i)}}{\partial \rho}+\frac{1}{\rho} H_{\phi(i)}(\rho, z)\right] .
$$

The radial eigenfunctions in the coaxial line that satisfy the correct boundary conditions for $T M_{0 n}$ modes on the inner and outer conductors are 


$$
\begin{aligned}
R_{n}(\rho) & \\
= & D_{0} / \rho \text { for } \mathrm{n}=0 \quad(\mathrm{TEM} \text { mode }) \\
= & \underbrace{D_{n}\left[J_{1}\left(k_{3 n} \rho\right) N_{0}\left(k_{3 n} a\right)-N_{1}\left(k_{3 n} \rho\right) J_{0}\left(k_{3 n} a\right)\right] \mathrm{n}>0}_{T M_{\text {on }} \text { modes }} .
\end{aligned}
$$

where $N_{n}$ are the Bessel functions of the second kind and the constants $D_{n}$ are obtained by requiring orthogonality

$$
\int_{a}^{b} \zeta R_{n}(b \zeta) R_{m}(a \zeta) d \zeta=\delta_{m n} \quad \mathrm{~m}, \mathrm{n}=0,1,2 \ldots
$$

Therefore

$$
D_{0}=\frac{1}{\sqrt{\ln (b / a)}}
$$

and

$$
D_{n}=\sqrt{\frac{1}{\int_{a}^{b} \rho R_{n}^{2}(\rho) d \rho}}=\frac{\pi k_{3 n}}{\sqrt{2}} \frac{1}{\sqrt{\frac{J_{0}^{2}\left(k_{3 n} a\right)}{J_{0}^{2}\left(k_{3 n} b\right)}-1}} .
$$

Unless stated otherwise we will assume that the radial eigenfunctions are normalized. The eigenvalues $k_{3 n}$ are the $n t h$ solutions of

$$
\left[J_{0}\left(k_{3 n} a\right) N_{0}\left(k_{3 n} b\right)-N_{0}\left(k_{3 n} a\right) J_{0}\left(k_{3 n} b\right)\right]=0 .
$$

The radial eigenfunctions are orthogonal over $[b, a]$ with respect to weight function $\rho$. The $T M_{0 n}$ propagation constants are

$$
\gamma_{i n}=j \sqrt{\left(\omega / c_{v a c}\right)^{2} \epsilon_{R(i)}^{*} \mu_{R(i)}^{*}-k_{i n}^{2}},
$$

the TEM mode propagation constant is

$$
\gamma_{i 0}=\frac{j \omega}{c_{v a c}} \sqrt{\epsilon_{R(i)}^{*} \mu_{R(i)}^{*}} .
$$

The electric field in the coaxial line is

$$
E_{\rho(3)}=R_{0}(\rho) \exp \left(-\gamma_{30} z\right)+\sum_{n=0}^{\infty} \Gamma_{3 n} R_{n}(\rho) \exp \left(\gamma_{3 n} z\right),
$$


and the magnetic field is

$$
H_{\phi(3)}=\frac{j \omega \epsilon_{3}}{\gamma_{30}} R_{0}(\rho) \exp \left(-\gamma_{3} z\right)-\sum_{n=0}^{\infty} \frac{j \omega \epsilon_{3}}{\gamma_{3 n}} \Gamma_{3 n} R_{n}(\rho) \exp \left(\gamma_{3 n} z\right) .
$$

In the shield region we assume we have evanescent $T M_{0 n}$ modes. The radial eigenfunctions in this region satisfy the condition $E_{z}=0$ on the boundary. The radial eigenfunctions must be finite at $\rho=0$, therefore Neumann function are not possible solutions. The eigenfunctions are

$$
G_{n}(\rho)=B_{n} J_{1}\left(\lambda_{n} \rho\right),
$$

where $B_{n}$ are normalization constants given by

$$
B_{n}=\frac{1}{\sqrt{\int_{0}^{b} \rho J_{1}^{2}\left(\lambda_{n} \rho\right) d \rho}}
$$

The fields can be written as

$$
E_{\rho(4)}=\sum_{n=1}^{\infty} D_{n} G_{n}\left(\lambda_{n} \rho\right) \exp \left(-\gamma_{4 n} z\right)
$$

and

$$
H_{\phi(4)}=\sum_{n=1}^{\infty} D_{n} \frac{j \omega \epsilon_{4}}{\gamma_{4 n}} G_{n}\left(\lambda_{n} \rho\right) \exp \left(-\gamma_{4 n} z\right)
$$

The eigenvalues $\lambda_{n}$ are found from eq (A.7) by letting $a \rightarrow 0$ so that

$$
J_{0}\left(\lambda_{n} b\right)=0
$$

Therefore $\lambda_{n}=p_{n} / b$ where $p_{n}$ is the $n$th root of $J_{0}(x)=0$.

The tangential component of the electric field is continuous across the coaxial line-shield interface at $z=0$. Matching eqs (A.10) and (A.14) at $z=0$ yields

$$
D_{n}=<G_{n} R_{0}>+\sum_{m=0}^{\infty}<G_{n} R_{m}>\Gamma_{m}
$$

where

$$
<G_{n} R_{m}>=\int_{a}^{b} \rho G_{n}(\rho) R_{m}(\rho) d \rho
$$


The tangential magnetic field components are also continuous across the coaxial line-shield interface. Matching eqs (A.11) and (A.15) at $z=0$ yields

$$
D_{n}=\frac{\gamma_{4 n}}{\gamma_{30}} \frac{\epsilon_{3}}{\epsilon_{4}}<G_{n} R_{0}>-\sum_{m=0}^{\infty} \frac{\epsilon_{3} \gamma_{4 n}}{\epsilon_{4} \gamma_{3 m}} \Gamma_{m}<G_{n} R_{m}>\text {. }
$$

Equations (A.17) and (A.19) yield

$$
<G_{n} R_{0}>+\sum_{m=0}^{\infty}<G_{n} R_{m}>\Gamma_{m}=\frac{\gamma_{4 n} \epsilon_{3}}{\gamma_{3 n} \epsilon_{4}}<G_{n} R_{0}>-\sum_{m=0}^{\infty} \Gamma_{m}<G_{n} R_{m}>\frac{\gamma_{4 n} \epsilon_{3}}{\gamma_{3 m} \epsilon_{4}}
$$

or

$$
\sum_{m=0}^{\infty} \underbrace{\left(1+\frac{\gamma_{4 n} \epsilon_{3}}{\gamma_{3 m} \epsilon_{4}}\right)<G_{n} R_{m}>}_{Q_{n m}} \Gamma_{m}=\underbrace{\left(\frac{\gamma_{4 n} \epsilon_{3}}{\gamma_{3 n} \epsilon_{4}}-1\right)<G_{n} R_{0}>}_{P_{n}}
$$

or

$$
\sum_{m} Q_{n m} \Gamma_{m}=P_{n}
$$

Only the reflection coefficient of the TEM mode $\left(\Gamma_{0}\right)$ is of interest since the other modes are evanescent in the coaxial line. 




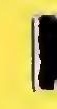

I

1

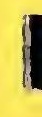

I

1

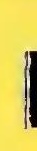

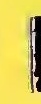

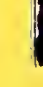

